
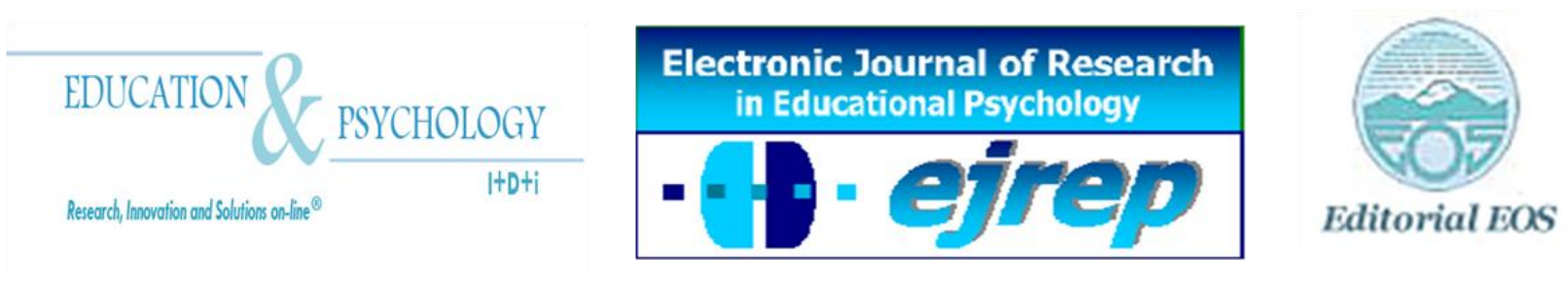

\title{
Un Modelo Instruccional para Guiar la Reflexión y la Investigación en el Aula: El Modelo de Calidad de Situación Educativa
}

Fernando Doménech Betoret

Developmental and Educational Psychology, Universitat Jaume I, Castellón

España

Dr. Fernando Doménech Betoret, Psicología Evolutiva y de la Educación. Universitat Jaume I, 12071 Castellón, España. E-mail: betoret@psi.uji.es. Tel.: +34 964/729550, Fax: +34 964/729262.

(C) Education \& Psychology I+D+i and Editorial EOS (Spain) 


\section{Resumen}

El propósito de este trabajo es presentar el Modelo de Calidad de Situación Educativa (MCSE), y cómo el profesorado puede utilizarlo para investigar y reflexionar en la situación educativa formal. Es un modelo teórico que trata de explicar de forma coherente el funcionamiento de una situación educativa formal, organizando y analizando las principales variables que intervienen en el aprendizaje escolar, así como las relaciones que mantienen entre ellas. Son escasos los modelos de situación educativa que aporten una vía metodológica para investigar en el contexto de la clase; sin embargo, el MCSE, además de ser un modelo conceptual, ofrece una vía metodología capaz de aportar datos para mejorar el proceso de enseñanza/aprendizaje y el rendimiento escolar. En este sentido, esta herramienta ha sido creada con el objetivo principal de guiar las investigaciones que se desarrollan en el contexto del aula e introducir al profesorado en la investigación psicoeducativa. Sólo obteniendo datos y evidencias de la situación educativa real se podrán iniciar acciones efectivas de mejora ajustadas a la realidad en la que se trabaja. En este trabajo se explican las características y funcionamiento del Modelo, y se ofrecen pautas e instrumentos para que cualquier profesor, tanto universitario como no universitario, pueda utilizarlo para mejorar la calidad del aprendizaje y el rendimiento de sus estudiantes.

Palabras clave: Modelo instruccional, marco conceptual, investigación en el aula, profesor reflexivo, situación educativa. 


\title{
An Instructional Model for Guiding Reflection and Research in the Classroom: The Educational Situation Quality Model
}

\begin{abstract}
The purpose of this work is to present an instructional model entitled the Modelo de Calidad de Situación Educativa" (MCSE) and how teachers can use it to reflect and investigate in a formal educational setting. It is a theoretical framework which treat to explain the functioning of an educational setting by organizing and relating the most important variables which according to the literature contribute to student learning. There are scarce educativional setting model that provide a methodological way to investigate in the educativional setting, however, the MCSE, besides to be a conceptual model, offers a methodology and instruments to obtain data in order to improve the teaching/learning process and academic achievement. Hence, the main objective of this tool is to guide the research conducted by teachers in the classroom and encourage them to investigate in the educational setting context. In this work, characteristics and functioning of the model are explained; moreover instructions and measure instruments are offered in order to be used by any teacher interested in improving the quality of student learning and achievement.
\end{abstract}

Key words: Instructional model, theoretical framework, research in the classroom, reflexive teaching, educational setting. 


\section{Introducción}

Los modelos educativos e instruccionales que existen hasta la fecha son bastante numerosos y tienen como principal propósito el ofrecer un marco conceptual para guiar la investigación en contextos formales (Anderson \& Burns, 1989). Sin embargo, son pocos los que proporcionan una vía metodológica para investigar en la situación educativa y que consideren el proceso de enseñanza y aprendizaje (en adelante E/A) de forma integrada, considerando de forma simultánea tanto lo que hace el profesor para enseñar como lo que hace el alumno para aprender.

Tras una exhaustiva revisión de la literatura (Doménech, 1991, 1995; Rivas \& Descals, 2000) de los principales modelos de situación educativa existentes, Doménech (2006, 2007, 2011a, 2011b, 2012) diseñó un nuevo modelo instruccional denominado Modelo de Calidad de Situación Educativa (MCSE). Aunque este modelo tiene algunas semejanzas con otros modelos causales, tales como el modelo 3P de Biggs (1978), modelo DEDEPRO (De la Fuente y Justicia, 2007, De la Fuente, 2011; Dunkin \& Biddle, 1974; Huitt, 2003; Pascarella, 1985), o el modelo CIPP (Stafelbeam y Skinkfild, 1989), posee características distintivas propias que lo hacen diferente. Entre las mismas, destacamos las siguientes: a) es un modelo integrado porque considera de forma simultánea los tres elementos clave de la instrucción (profesor, estudiante, contenido/curriculum) cuyas características de partida o input van a condicionar desde el principio el resultado de la instrucción; b) secuencial, porque las tres fases (input, proceso y cambio) que componen el modelo se desarrollan de forma secuencial hasta completar un ciclo instruccional (tema, unidad instruccional, bloque temático, etc.); c) sistémico porque opera como un sistema, ya que además de estar sus elementos interrelacionados, posee capacidad de autorregulación propia para alcanzar los objetivos propuestos; d) no es solamente un modelo conceptual sino que aporta una vía metodológica e instrumentos de medida para obtener datos orientados a mejorar el proceso de E/A que tiene lugar en una SE formal.

El Modelo de Calidad de Situación Educativa (a partir de ahora MCSE) se muestra en las figuras 1 y 2. La figura 1 ofrece una visión global de su funcionamiento, mostrando la actuación simultánea e integrada del profesor y estudiantes. El modelo se expande en la figura 2, donde el proceso se desglosa en dos nuevas fases o subfases: la fase inicial de 
posicionamiento y la fase de implicación interactiva. La figura 2 muestra además las relaciones funcionales entre todos los componentes del modelo.

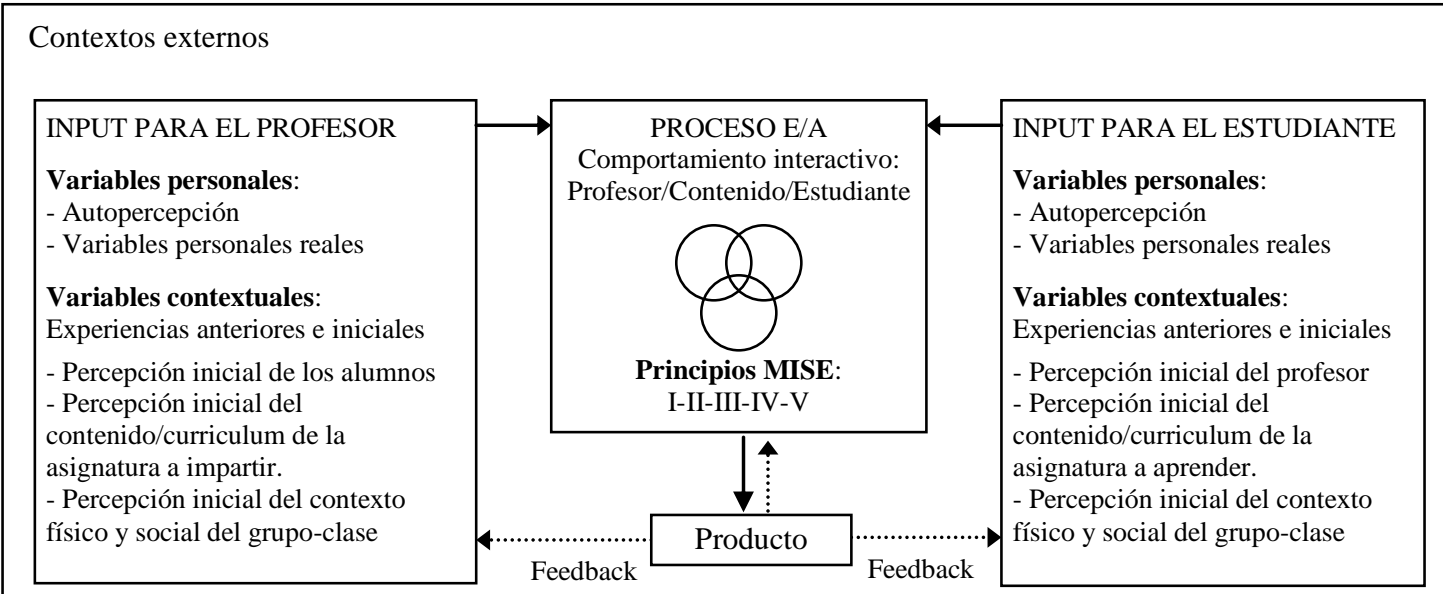

Figura 1. Modelo de calidad de Situación Educativa (MCSE): Interrelación entre input, proceso y producto.

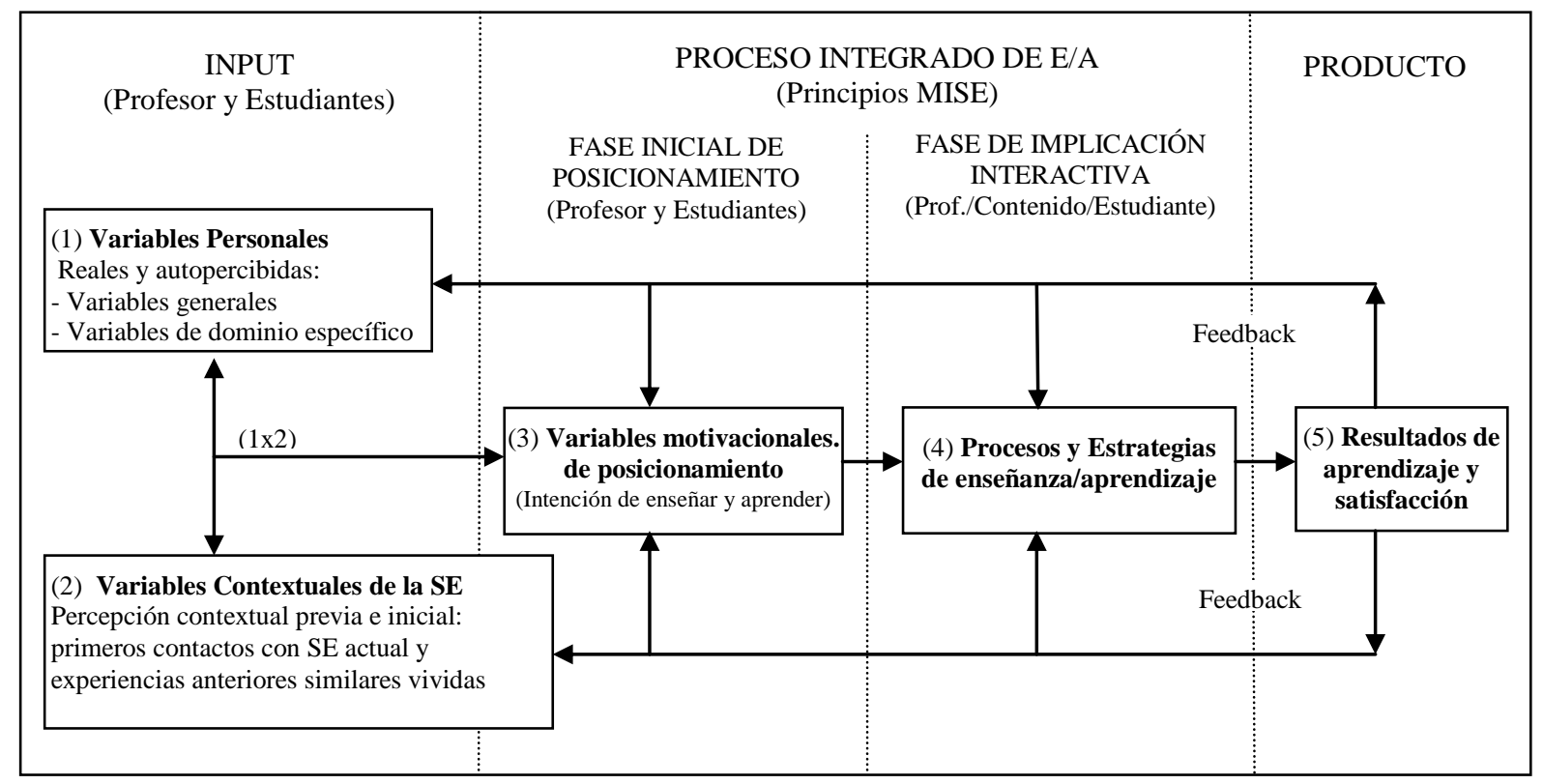

Figura 2. Modelo de calidad de Situación Educativa (MCSE): Estructura, componentes y relaciones.

Como se puede ver en la figura 2, el modelo está compuesto por cinco bloques de variables, distribuidas en tres fases secuenciales que determinan el producto o rendimiento alcanzado por el estudiante. La fase input está formado por el bloque 1 (variables personales) 
y el bloque 2 (variables contextuales). La fase de proceso, esta formada por el bloque 3 (variables motivacionales de posicionamiento) y el bloque 4 (Procesos y estrategias de enseñanza/aprendizaje) $\mathrm{y}$, finalmente, la fase de producto esta formada por el bloque 5 (resultados de aprendizaje). Todo su funcionamiento está condicionado por contextos externos, como los supracontextos (ej.: departamento, centro, sistema educativo vigente, etc.) y contextos paralelos (ej.: la familia). A continuación pasamos a comentar las fases y componentes del modelo:

\section{Fase input}

El componente input responde a¿qué elementos van a intervenir y en qué circunstancias con la intención de provocar aprendizajes?. En él se recogen todas aquellas variables de partida referidas tanto a las características personales de los agentes participantes (profesor, estudiantes) como a las condiciones contextuales iniciales que según la literatura influyen de forma importante en el aprendizaje del escolar. Las condiciones o circunstancias se refieren a aquellas variables que describen las características más destacadas de los contextos de aprendizaje (contexto de clase y contextos externos) percibidas por los agentes intervinientes (profesor y estudiantes) que van a condicionar el proceso de E/A desde el inicio.

La investigación psicológica ha constatado que la conducta de una persona está determinada por sus variables personales y ambientales. El modelo cognitivo de Lazarus y Folkman (1984), explicativo de como las personas responden ante una situación potencialmente estresante, profundiza en esta idea. Según estos autores las personas antes de actuar o responder ante un determinado problema o de enfrentarse a cualquier situación, hacen una evaluación de sus variables personales (evaluación primaria) y una evaluación de las características de la situación (evaluación secundaria). A través de la información que proporcionan dichas evaluaciones, el sujeto responderá, ante una situación potencialmente estresante, de una forma o de otra. Entendemos que la propuesta de Lazarus and Folkman (1984) puede ser válida para aplicarla al contexto de la clase, de modo que la motivación inicial del sujeto va a depender, en gran medida, del resultado de su evaluación primaria (variables personales) y de su evaluación secundaria (variables contextuales). En ese sentido, el modelo MCSE tiene en cuenta tanto las variables personales de los sujetos intervinientes en la instrucción como las variables contextuales. El nivel de motivación con que el profesor y 
los estudiantes afronten, desde el principio, el proceso de E/A se operacionaliza y concreta a través de lo que hemos denominado, las "Variables Motivacionales de Posicionamiento" (VMP) que van a determinar la "intención de aprender" del estudiante y la "intención de enseñar" del profesor.

\section{Variables personales (bloque 1)}

Las variables personales (bloque 1) están agrupadas en dos categorías: generales y específicas. Según Boekaerts (1999, p. 44) las variables personales generales indican "general feeling of competence or inclination to engage in scholastic learning". La medida de dichas variables "only describe properties that are common to a wide range of learning situations" (Boekaerts, 1999, p. 44). Las variables personales generales se caracterizan por su alta estabilidad y generalidad, abarcando variables tales como personalidad, inteligencia, aptitudes, estilos, etc.; o bien, dimensiones generales del sujeto (autoconcepto general, estilo atribucional, ansiedad como rasgo, etc.). Las variables de dominio específico indican la tendencia del estudiante a reaccionar de forma favorable o desfavorable en el aprendizaje de un contenido específico (Boekaerts, 1999). Estas son más inestables y emergen en situaciones educativas específicas. Esta categoría incluye variables tales como conocimientos previos sobre un dominio específico, interés en un dominio específico, etc.; o dimensiones personales específicas (ansiedad como estado, autoconcepto en matemáticas, etc.). Actualmente las variables personales con las que estamos trabajando son las siguientes: conocimientos previos, autoeficacia académica general, interés por la materia, creencias sobre el alcance formativo de la materia y autoestima.

\section{Variables contextuales de la SE (bloque 2)}

Las variables contextuales (bloque 2) se refieren a los primeros contactos que hayan tenido el profesor y estudiantes con la situación educativa actual, así como experiencias anteriores similares vividas. En este sentido, será importante considerar, desde el inicio de curso, cual ha sido la percepción (creencias, juicios, actitudes) que se ha formado el profesor de los estudiantes, del contenido a impartir y del escenario instruccional (contexto físico y social) ya que va a condicionar la forma de enseñar del profesor. "La percepción que realiza el profesor de las características de los alumnos (más o menos próximos culturalmente, más o menos diligentes en sus tareas, más o menos acomodaticios a las normas, más o menos brillantes en su aprendizaje), dan lugar a diferentes expectativas en el profesor y a unas formas diferentes de comportamiento interactivo con ellos" (Rosales, 2000, p. 47). Del mismo 
modo, también será importante conocer la percepción que se han formado los estudiantes del profesor, de los contenidos que tienen que aprender, de cómo serán evaluados sus aprendizajes y del escenario instruccional (contexto físico y social), ya que va a condicionar la forma de aprender del estudiante. Numerosos estudios previos han constatado (e.g. Doyle, 1977; Fraser, 1987, 1989, 1998; Ramsden, 1992; Waxman, 1991) que las percepciones generadas por los estudiantes del entorno de aprendizaje tienen una notable influencia en la calidad del aprendizaje y en los resultados académicos obtenidos. La importancia que se concede a la interpretación de la situación por la gente que participa en ella, se debe a que las personas implicadas en dicha situación actúan según la percepción que tienen de dicha situación (Erickson, 1986). El contexto social de cada situación educativa es diferente, debido a que genera diferentes percepciones en el profesor y en los estudiantes, y a las actuaciones que se derivan de tales percepciones (Erickson, 1986). Basándonos en estas consideraciones teóricas se elaboró la escala (Doménech, 2007) orientada a evaluar la percepción generada por el estudiante al inicio del curso, y que ha sido recientemente revisada (ver Doménech, 2011, 2012).

\section{Fase de proceso}

El punto de partida para identificar y organizar las variables de proceso ha sido el Modelo Instruccional de Situación Educativa (MISE) Rivas (1993, 1997, 2003). Dicho modelo esta formado por 5 dimensiones (denominadas principios por el profesor Rivas) que organizan de forma secuencial y jerárquica todo el proceso de E/A desarrollado con un contenido curricular específico: P1. Intencionalidad (objetivos y motivación escolar), P2. Diseño de instrucción, P3. Interacciones personales, P4. Adquisición de conocimientos, P5. Control y evaluación. Así, la instrucción parte del P1 o principio de Intencionalidad (metas y objetivos), que activa el proceso educativo y se mantiene mientras dura. La planificación para lograr las metas y objetivos educativos implica la puesta en funcionamiento del principio siguiente, P2 (diseño de Instrucción). El desarrollo e implementación del diseño en el aula implica la existencia de P3 (interacciones personales) mientras se logra P4 (adquisición de conocimientos), y finalmente actúa el principio P5 (control y evaluación). El P5 produce retroalimentación a los demás principios instruccionales que le anteceden. La conducta desplegada por el profesor y el alumno con respecto a un contenido curricular específico, o asignatura, se desarrollaría de forma interactiva a lo largo de los 5 principios o dimensiones del MISE, en donde el profesor y el estudiante jugarían un nivel diferente de responsabilidad. 
Así, como se puede ver en la Tabla 1, el principio P1 (intencionalidad) es de responsabilidad compartida entre el profesor y los estudiantes, el P2 (diseño de la instrucción) es de responsabilidad fundamental del profesor, el P3 (interacciones personales) es de responsabilidad compartida, el P4 (adquisición de conocimientos) es de responsabilidad fundamental del estudiante y, finalmente, el P5 (Evaluación) es de responsabilidad principal del profesor. Cada principio o dimensión incluye un conjunto de indicadores (ver Tabla 1) que operacionalizan y concretan las acciones que se dan en cada principio entre los elementos clave (profesor/contenido/estudiantes). Los indicadores que concretan cada principio han sido seleccionados a partir de una revisión detallada y exhaustiva de la literatura especializada (Gómez, 1993; Martínez, 1991, 1995; Doménech, 1991, 1995; Descals, 1996).

Tabla 1. Principios e Indicadores del MISE y elemento clave (profesor/contenido/estudiante) en el que recae la responsabilidad principal de cada indicador.

\begin{tabular}{|c|c|c|c|}
\hline P. I. INTENCIONALIDAD : Objetivos y motivación escolar & Profesor & Contenido & Estudiante \\
\hline I. 1.1. Cambio de estado en el aprendiz. & $\mathrm{x}$ & $\mathrm{X}$ & $\mathrm{x}$ \\
\hline I. 1.2. Expectativas iniciales & $\mathrm{X}$ & $\mathrm{x}$ & $\mathrm{x}$ \\
\hline I. 1.3. Significación personal. & $\mathrm{X}$ & $\mathrm{x}$ & $\mathrm{x}$ \\
\hline \multicolumn{4}{|l|}{\begin{tabular}{|l} 
P. II. DISEÑO INSTRUCTIONAL: Planificación del proceso E/A. \\
\end{tabular}} \\
\hline I. 2.1. Estructuración de contenidos, actividades y control. & $\mathrm{X}$ & $\mathrm{x}$ & \\
\hline I. 2.2. Estrategias de enseñanza. & $\mathrm{X}$ & $\mathrm{x}$ & \\
\hline I. 2.3. Logística de recursos didácticos. & $\mathrm{X}$ & $\mathrm{x}$ & \\
\hline I. 2.4. Temporalidad expositiva y condiciones físicas. & $\mathrm{X}$ & $\mathrm{x}$ & \\
\hline I. 2.5. Tácticas de individualización complementarias. & $\mathrm{X}$ & & \\
\hline \multicolumn{4}{|l|}{ P. III. INTERACCIONES PERSONALES: Clima de la clase. } \\
\hline I. 3.1. De primer nivel: Emisor/Aprendiz. & $\mathrm{X}$ & & $\mathrm{x}$ \\
\hline I. 3.2. De segundo nivel: Relaciones entre pares. & & & $\mathrm{x}$ \\
\hline I. 3.3. De tercer nivel: Relaciones funcionales sintagmáticas & $\mathrm{x}$ & & \\
\hline \multicolumn{4}{|l|}{$\begin{array}{l}\text { P. IV. ADQUISICIÓN DE CONOCIMIENTOS: Procesos de } \\
\text { aprendizaje }\end{array}$} \\
\hline I. 4.1. Parámetros evolutivos: Condicionantes y activadores. & & & $\mathrm{x}$ \\
\hline I. 4.2. Conocimientos previos: Concepciones y contenidos. & & $\mathrm{x}$ & $\mathrm{x}$ \\
\hline I. 4.3. Conocimientos: Declarativos y procedimentales. & & $\mathrm{x}$ & $\mathrm{x}$ \\
\hline I. 4.4. Procesos atencionales y sistema de representación. & & $\mathrm{x}$ & $\mathrm{x}$ \\
\hline I. 4.5. Estrategias, estilos y tareas de aprendizaje. & & $\mathrm{x}$ & $\mathrm{x}$ \\
\hline I. 4.6. Diferencias individuales: Capacidades. & & & $\mathrm{x}$ \\
\hline I. 4.7. Parámetros temporales: Dedicación & & & $\mathrm{x}$ \\
\hline \multicolumn{4}{|l|}{$\begin{array}{l}\text { P. V. CONTROL Y EVALUACION: Retroalimentación del } \\
\text { proceso E/A. }\end{array}$} \\
\hline I. 5.1. Control y evaluación durante el proceso de E/A: Formativa. & $\mathrm{X}$ & $\mathrm{x}$ & \\
\hline I. 5.2. Control y evaluación posterior al proceso E/A: Final. & $\mathrm{X}$ & $\mathrm{x}$ & \\
\hline I. 5.3. Efecto psicológico individual: Ansiedad/Estrés. & $\mathrm{X}$ & & \\
\hline
\end{tabular}

El componente proceso, inicialmente estructurado según los 5 Principios o dimensiones del MISE, en la última revisión del modelo que nos ocupa, se ha desglosado en dos grandes bloques de variables (bloque 3 y bloque 4) organizados secuencialmente. Esta 
restructuración responde a una doble finalidad: a) captar dos momentos importantes del proceso educativo y b) simplificar y hacer más parsimonioso el MCSE. Así, el bloque 3 denominado "variables motivacionales de posicionamiento" se corresponde a la "fase inicial de posicionamiento" y el bloque 4 denominado "Procesos y estrategias de enseñanza/aprendizaje" se corresponde con la "fase de implicación interactiva". El bloque 3 tiene su equivalencia con primer principio (P1) del MISE, ya que las variables que lo integran se refieren a la intencionalidad y motivación inicial del profesor y de los estudiantes para enseñar y aprender unos contenidos específicos. Mientras que el bloque 4 engloba los restantes Principios o dimensiones del MISE: P2 (Diseño de instrucción), P3 (Interacciones personales), P4 (Adquisición de conocimientos) y P5 (Evaluación). A continuación comentaremos con más detalle cada uno de estos dos bloques que conforman la fase de proceso del MCSE.

\section{Variables Motivacionales de Posicionamiento (bloque 3)}

Se refieren a aquellas variables personales pertenecientes al componente afectivomotivacional, que se van a generar o activar en los sujetos participantes (motivación inicial, expectativas, actitudes, orientación de meta, etc.) durante los días previos e iniciales al proceso instruccional en función de la información que reciban sobre el desarrollo del proceso de E/A y de sus variables personales. Es como la primera imagen que te formas de una persona y que después resulta difícil cambiar. Como podemos ver en la figura 2 estas variables ocupan un lugar intermedio entre el input y el proceso propiamente dicho pero tienen cierto solapamiento en el tiempo con las variables contextuales del bloque 2, aunque las variables de posicionamiento son una consecuencia de ellas. Es una fase de preproceso que hemos denominado «fase inicial de posicionamiento», por ser variables personales que se generan al inicio del proceso educativo.

Son variables que se activan en la fase inicial del proceso de E/A en función de la percepción que los tengan los estudiantes del profesor, contenido/curriculum (metodología y evaluación) y clima social de la clase (bloque 2), y que determinan la intención de enseñar del profesor y la intención de aprender del estudiante. Si bien, el grado de activación de estas variables estará modulado por ciertas variables de tipo personal (bloque 1). Las variables motivacionales de posicionamiento (VMP) actúan como una ola que recorre todo el proceso de E/A hasta que éste finaliza, y durante ese recorrido pueden fortalecerse o debilitarse en 
función de que mejore o empeore la percepción de los elementos anteriormente señalados (Doménech, 2012). Aunque, al igual que sucede con la primera impresión que nos formamos de una persona, lo más probable es que si la activación de las variables motivacionales de los estudiantes es alta al inicio de un proceso educativo, termine alta, y si la activación es baja termine baja (Doménech, 2012).

\section{Variables Motivacionales de Posicionamiento (VMP) generadas en el estudiante}

Las variables generadas en el aprendiz responden a su forma de percibir, o a la idea que se haya formado, de cómo se va a desarrollar el proceso instruccional sobre unos contenidos concretos y un profesor determinado. Esta idea se puede ir gestando, bien antes de iniciarse el proceso de E/A, por las experiencias anteriores que han tenido con ese tipo de contenido, por la información que conoce de ese profesor, por lo que le han dicho compañeros más veteranos, etc.; o bien durante los primeros días de clase, al conocer al profesor, el programa de la asignatura, cuáles serán sus demandas, la presentación que ha hecho el profesor de cómo se va a trabajar la materia, etc. La percepción que se haga el alumno/a, durante esos días previos e iniciales, sobre cómo va a ser el proceso de E/A desarrollado con esa materia y con ese profesor (cómo va a ser la actuación de ese profesor, la dificultad y utilidad de la materia, el grado de exigencia para superar la materia, etc.) van a condicionar desde el principio su forma de afrontar ese proceso (implicación, dedicación, esfuerzo, etc.) y en consecuencia su aprendizaje.

Efecto de la interacción (1x2) en el estudiante: La interacción de estos dos componentes determinan su "intención de aprender". La intención de aprender se genera a partir de ciertos procesos y reflexiones mentales que se concretan en las siguientes cuestiones implícitas: ¿Tendré éxito en esta materia?, Qué valor tiene esta asignatura para mí? y ¿Cómo me sentiré en esta asignatura? (adaptados de Pintrich, 1989; Pintrich y De Groot, 1990). A les tres preguntas anteriores podríamos añadir una cuarta: ¿Cuánto tiempo y esfuerzo tendré que dedicar a esta materia impartida por este profesor? (derivada de la Teoría de la expectancia de Vroom, 1964). Estas cuestiones se han tenido en cuenta para elaborar la escala orientada a evaluar la intención de aprender del alumno/a.

\section{Variables Motivacionales de Posicionamiento (VMP) generadas en el profesor}

El profesor también se va a formar su propia idea personal de cómo se va a desarrollar el proceso de E/A con el contenido concreto que va a impartir a unos estudiantes 
determinados. Antes de empezar, por las experiencias anteriores que ha tenido en similares situaciones educativas, y durante los primeros días, mientras va conociendo las características de los estudiantes, personalmente o a través de la información proporcionada por sus compañeros del centro. La percepción que se forme el profesor durante esos días previos e iniciales, sobre cómo va a ser el proceso de E/A que se va a desarrollar en esa situación educativa, con esos estudiantes, va a condicionar poderosamente, desde el principio, su forma de afrontar dicho proceso (implicación, dedicación, esfuerzo, etc.).

Efecto de la interacción (1x2) en el profesor: La interacción de estos dos componentes determinan su "intención de enseñar". La intención de enseñar se genera a partir de ciertos procesos y reflexiones mentales que se concretan en las siguientes cuestiones implícitas: ¿Tendré éxito enseñando esta materia a estos alumnos/as? (se refiere a los objetivos de aprendizaje que tiene que alcanzar), ¿Qué reto, valor o importancia tiene para mí como profesor alcanzar los objetivos propuestos en esta asignatura con estos estudiantes?, ¿Cómo me sentiré enseñando esta asignatura? (adaptado de Pintrich, 1989; Pintrich \& De Groot, 1990).Y finalmente ¿Cuánto tiempo y esfuerzo tendré que dedicar a alcanzar los objetivos de esta materia con estos estudiantes? (derivada de la Teoría de la expectancia de Vroom, 1964). Estas cuestiones serán el punto de partida para elaborar la escala (actualmente en proceso) orientada a evaluar la intención de enseñar del profesor/a.

\section{Teorías que sustentan las Variables Motivacionales de Posicionamiento}

Las Variables Motivacionales de Posicionamiento (VMP), agrupadas en el bloque 3, y las escalas construidas para evaluar dichas variables, se derivan de aquellas teorías motivacionales clásicas que tratan de explicar cómo funciona la motivación inicial, es decir, aquella que nos indica si debemos abordar e implicarnos en una tarea o por el contrario renunciar a ella. A continuación pasamos a comentar brevemente dichas teorías:

Teoría de la expectativa-valor (Feather, 1982; Vroom, 1964)

Según la teoría de la expectativa-valor existen dos factores que hacen que un estudiante decida esforzarse para aprender una materia o no:

1. La importancia de la materia, que debe tener algún valor para el estudiante.

2. La probabilidad de tener éxito en el aprendizaje de dicha materia. 
En efecto, la experiencia nos dice que nadie inicia algo que crea que no merece la pena o que las oportunidades de éxito sean escasas. La realización de la tarea se consideraría en estas circunstancias una pérdida de tiempo. Por tanto, la motivación inicial para abordar una tarea dependerá del producto de estos dos factores, de modo que si uno de ellos es cero no se producirá motivación. Aunque el concepto de valor parece relativamente simple, no lo es tanto y sus determinantes son muchos Un objeto puede tener un valor intrínseco, extrínseco e instrumental (como un paso para conseguir un objetivo a más largo plazo)

\section{Teoría de la motivación del logro de Atkinson (1964)}

La motivación del logro se puede definir como "el deseo de conseguir el éxito". Los elementos constitutivos de la motivación de logro son: el motivo, la expectativa y el incentivo (valor o importancia de la meta). Atkinson señala que la conducta humana orientada al logro es el resultado del conflicto aproximación-evitación, es decir, la motivación para lograr el éxito y evitar el fracaso. Alguien tiene motivación de logro si el motivo «alcanzar el éxito» es mayor que el motivo «evitar el fracaso» Sólo si ocurre esto se sentirá el sujeto impulsado a actuar en persecución de una determinada meta. Por tanto, resulta que cuando el motivo de un sujeto para lograr el éxito es más fuerte que el motivo a evitar el fracaso, la tendencia resultante es positiva, y más fuerte cuando la tarea es de mediana dificultad.

Teoría motivacional de Pintrich, (1989), Pintrich y De Groot (1990):

Pintrich y De Groot encontraron que el valor intrínseco que se otorga a la actividad influye especialmente en la implicación inicial del sujeto en la tarea. Es también al comienzo de la actividad cuando el sujeto adopta una orientación de meta determinada (Pintrich, 2000) que se va adaptando e incluso modificando durante la fase de ejecución. El marco teórico sobre motivación planteado por los autores señalados está integrado por tres componentes principales. El componente de expectativa, que hace referencia a las creencias y expectativas de los estudiantes para realizar una determinada tarea (creencias de autoeficacia). Este componente se podría traducir en la siguiente pregunta: ¿soy capaz de hacer esta tarea?. El componente de valor, que indica las metas de los alumnos (orientación de meta) y sus creencias sobre la importancia e interés de la tarea. Este componente se podría traducir en la siguiente pregunta: ¿por qué hago esta tarea?. El componente afectivo, que recoge las reacciones emocionales de los estudiantes ante la tarea. Este componente se podría traducir en la siguiente pregunta: ¿cómo me siento al hacer esta tarea?. 


\section{Procesos y estrategias de enseñanzalaprendizaje (bloque 4)}

El bloque 4 correspondiente a la "fase de implicación interactiva". Esta fase trata de responder $a_{\complement} Q u e ́$ ha hecho el profesor para enseñar y el estudiante para aprender?. Se refiere a la forma de relacionarse y de interactuar los tres elementos clave: profesor, contenido y aprendiz como consecuencia del comportamiento generado por el profesor y los alumnos/as con un contenido curricular específico o asignatura. La conducta del profesor, ya sea como instructor o como gestor del grupo-clase, abarca todo lo que el profesor hace para que el alumno aprenda. La conducta del alumno abarca todo lo que éste hace para aprender, es decir, para dominar las competencias formuladas por el profesor, reflejadas en el programa de la asignatura y concretadas en objetivos de aprendizaje. A través de las estrategias de aprendizaje podemos procesar, organizar, retener, y recuperar la información, a la vez que planificamos, regulamos y evaluamos esos mismos procesos en función de los objetivos a alcanzar, determinados por las demandas de la tarea (Beltrán, 1993, 1998).

En base a estas consideraciones, el bloque 4 está formado por aquellas variables relativas a las estrategias de E/A desplegadas por el profesor y estudiantes para alcanzar los objetivos de aprendizaje prefijados. Pero, las estrategias no son únicamente las derivadas de acciones directas, sino que también implican procesos y metacogniciones que regulan el plan de acción de todo proceso educativo como autoanálisis, autoevaluación de lo realizado, etc. (Beltrán, 1993, 1998; Pozo, 1989), de ahí que en este bloque también se tengan en cuenta variables relativas a los procesos autorregulatorios y metacognitivos que llevan a cabo los profesores cuando enseñan y los alumnos cuando aprenden.

Para organizar y evaluar el bloque 4, al que hemos denominado "Procesos y estrategias de enseñanza/aprendizaje" nos hemos basado en los principios del modelo MISE (excepto en el P1 que pertenecería al bloque anterior): P2 (diseño de instrucción), P3 (implementación diseño: interacciones personales), P4 (adquisición de conocimientos), y P5 (evaluación). Ha partir de estas dimensiones y sus correspondientes indicadores (ver Tabla 1), hemos construido las escalas para evaluar el proceso de E/A de forma integrada, recientemente revisadas (MISE-R para el profesor y MISE-R para el estudiante) por Doménech (2011, 2012). Los cuestionarios MISE-R evalúan el proceso de E/A de forma global e integrada, no obstante al investigador le puede interesar centrar su atención en algún/algunos principio/s e indicador/es determinados (por ejemplo aquellos que son de 
responsabilidad fundamental del estudiante), en ese caso, a modo de zoom, puede dirigir su foco sobre dicho principio/indicadores y amplificarlos construyendo una escala más detallada orientada a evaluar solamente el/los principio/s e indicador/es de interés.

Fase producto: Resultadosy satisfacción (bloque 5)

El producto de la instrucción responde a ¿Qué se ha logrado o conseguido? Y hace referencia a los resultados de aprendizaje alcanzados por el estudiante (learning outcomes). El aprendizaje lo entendemos como un cambio producido en el estudiante al pasar de un estado inicial a un estado final. Tradicionalmente, en la universidad se ha buscado provocar cambios en los estudiantes de tipo informativo o cuantitativo-conceptual en lugar de cambios formativos más útiles y eficaces para resolver problemas y tomar decisiones. Sin embargo con el cambio de paradigma pedagógico impulsado desde el Espacio Europeo de Educación Superior (EEES) se pretende caminar en esta dirección, para ello se ha redefinido el tipo de producto que se pretende alcanzar en términos de competencias genéricas y específicas, más acorde con los retos y desafíos que plantea la sociedad actual del conocimiento. Así pues, el cambio formativo se identifica con el aprendizaje que queremos conseguir en nuestros estudiantes formulados en términos de competencias y operacionalizados a través de resultados de aprendizaje. Finalmente señalar que en este bloque, además de los resultados, también se tiene en cuenta la satisfacción experimentada tanto por el profesor como por los estudiantes, ya que es importante que el profesor disfrute enseñando y el estudiante disfrute aprendiendo.

\section{¿Cómo utilizar el modelo para reflexionar e investigar en la situación educativa formal?}

La mayoría de los autores consideran que el desarrollo de la práctica reflexiva es la base para conseguir unas altas competencias docentes (Cole \& Knowles, 2000; Jay, 2003; Larrivee, 2000; Osterman \& Kottkamp, 2004; Zeichner \& Liston, 1996). Los programas de formación que se están impulsando actualmente tanto para profesores universitarios como no universitarios tratan de construir un nuevo tipo de profesional práctico-reflexivo, que posee conocimientos que aplica a la práctica y que reflexiona sobre los resultados de su acción. Desde esta perspectiva los profesores son sujetos que realizan sus propias investigaciones en el contexto de la clase (plantear hipótesis, seleccionar los instrumentos de medida, recoger, analizar e interpretar los datos, etc.) para mejorar sus prácticas y perfeccionarse (Schön, 1992; 
Zeichner, 1993). Los estudios que se han realizado en este contexto indican que los profesores que investigan su propia práctica se muestran más satisfechos con su profesión, sobre todo porque la investigación les ayuda a comprender y transformar sus prácticas escolares (Cochram-Smith \& Lytle, 1993).

El modelo MCSE proporciona un marco conceptual y la instrumentación necesaria para facilitar la reflexión del docente y guiar la investigación en una situación educativa concreta, tanto a nivel universitario como no universitario. Las escalas proporcionadas son capaces de identificar los puntos fuertes y débiles del proceso de E/A seguido en la clase a través de la participación simultánea de los tres elementos clave (profesor, contenido, estudiantes). Ello facilitará la reflexión del profesor sobre su propia práctica pedagógica y sus consecuencias, y permitirá introducir las correcciones oportunas para mejorar la docencia y el aprendizaje. Los instrumentos aportados también van a permitir evaluar y diagnosticar los "factores pronóstico" y determinar el papel que pueden jugar en una situación educativa concreta.

Los componentes input y de posicionamiento constituyen los "factores pronóstico" del estudiante y del profesor, de modo que las variables que los conforman nos pueden ayudar a pronosticar el producto o resultados a alcanzar por los estudiantes. Especificando un poco más podríamos decir, aplicando terminología médica, que una puntuación baja en las "variables motivacionales de posicionamiento" denotarían los síntomas de una enfermedad denominada "amotivación" (ver teoría de la autodeterminación de Deci \& Ryan, 1985), y las causas desencadenantes de dicha falta de motivación habría que buscarlas en las variables input. Aunque los resultados todavía no son concluyentes, la investigación realizada hasta la fecha (Doménech, 2006, Doménech, 2011b) parece indicar que las variables motivacionales de posicionamiento tienen una importante capacidad predictiva sobre la forma de aprender del estudiante y sobre el rendimiento escolar. En este sentido queremos enfatizar la importancia de evaluar estas variables en los estudiantes, al inicio del curso, porque aportarán información valiosa al profesorado de como los estudiantes van a afrontar el proceso de E/A que se va a iniciar con una determinada asignatura. Conocer dicha información al principio de curso permitirá intervenir a tiempo cuando la situación lo requiera e implementar acciones correctoras desde el principio para aquellos estudiantes que presenten un mal pronóstico de partida. En la Figura 3 se pueden ver las diferentes formas que pueden evolucionar las variables motivacionales de posicionamiento a lo largo del proceso de E/A. 


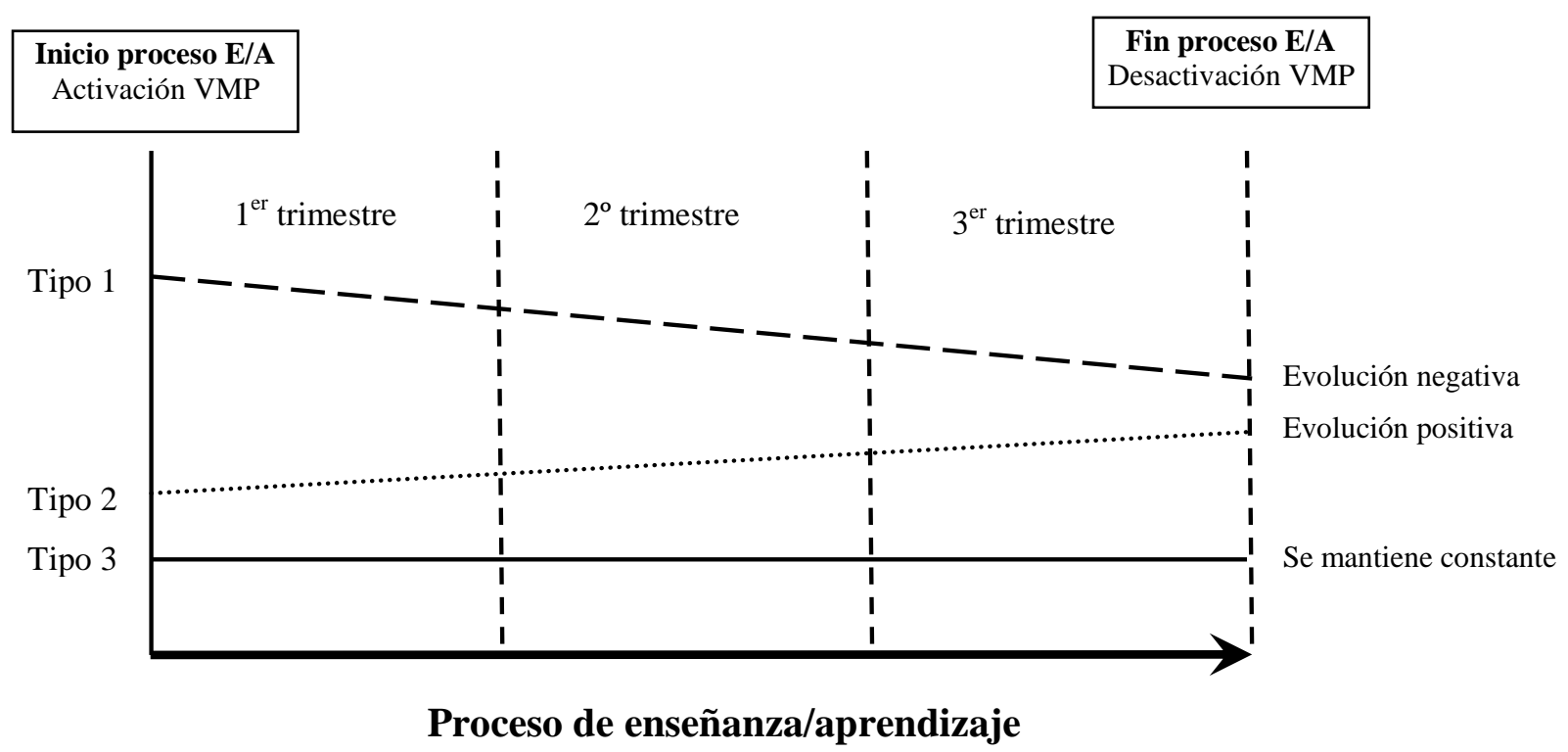

Figura 3. Modos en que puede evolucionar la motivación inicial (VMP) durante el proceso de E/A.

Basándonos en los planteamientos expuestos, a continuación, vamos a formular algunas preguntas de investigación interesantes para que cualquier profesor comprometido pueda aplicarlas a su situación educativa concreta, y obtener, así, datos científicos que le permitan, desde la reflexión, mejorar la práctica docente y el aprendizaje de los alumnos.

- ¿Cómo ha evolucionado motivación inicial de los alumnos (intención de aprender) desde el inicio del desarrollo del tema/asignatura hasta su finalización?

- ¿Cómo ha contribuido, en el nivel motivacional de los alumnos, el proceso de E/A desarrollado para alcanzar los objetivos programados? ¿Cuáles han sido las variables que más y menos han influido?

- ¿Cuál ha sido el grado de predicción de la motivación inicial de los alumnos (intención de aprender) sobre el rendimiento y satisfacción de los alumnos.

- ¿Por qué unos alumnos están más motivados que otros para iniciar el estudio de un tema o asignatura concreta? ¿Qué capacidad explicativa de la motivación inicial o intención de aprender tienen las variables personales del alumno?

- ¿Qué capacidad explicativa de la motivación inicial o intención de aprender tiene la percepción inicial que se forma el alumno sobre las variables contextuales de la clase, es decir, del profesor, del contenido/curriculum y de los compañeros?

La evaluación del producto (resultados alcanzados y satisfacción experimentada por el profesor y los estudiantes) nos permitirá comprobar la calidad de los aprendizajes alcanzados 
(operacionalizados a través de los objetivos de aprendizaje) y, a la vez, nos proporcionará feedback sobre cómo se han comportado los componentes del modelo, intervinientes, directa o indirectamente, en al producto final obtenido. Unos malos resultados, acompañados de poca satisfacción respecto al proceso seguido, por parte del profesor y/o de los alumnos, significaría que uno o varios componentes del modelo han funcionado de forma deficiente y convendría revisarlos.

Para concluir, se destaca que los instrumentos desarrollados, hasta la fecha, para evaluar cada uno de los componentes del MCSE se pueden consultar y descargar en la siguiente dirección web: http://www3.uji.es/ betoret/

Cuestionario MCSE-variables personales del estudiante permitirá evaluar algunas de las características personales del estudiante que han demostrado tener una notable influencia en su motivación inicial o intención de aprender. La aplicación de este instrumento se debería realizar al inicio del curso.

Cuestionario MCSE-percepción inicial del estudiante permitirá evaluar la percepción o idea inicial que se forme el estudiante de cómo se va a desarrollar el proceso de E/A con un contenido escolar específico y un profesor determinado. La aplicación de este instrumento se debería realizar al inicio del curso, tras varios días de clase, para dar tiempo a los estudiantes a que se formen su propia idea del proceso instruccional que se va a desarrollar.

Cuestionario MCSE-posicionamiento del estudiante posibilitará la evaluación de las variables motivacionales del aprendiz, determinantes de la intención de aprender. La aplicación de este cuestionario, al igual que el anterior, se debería realizar al inicio de curso tras varios días de clase, para dar tiempo a los estudiantes a que se formen su propia idea del proceso instruccional que se va a desarrollar; ya que esta idea inicial, modulada por las variables personales, generarán su intención de aprender.

Cuestionarios MISE-R para el profesor y MISE-R para el estudiante nos permitirá evaluar, el proceso de E/A desarrollado con una asignatura específica, desde el punto de vista del profesor y de los estudiantes, lo que permitirá contrastar la información proporcionada por el profesor y los estudiantes con respecto a unos mismos referentes (los principios o dimensiones del MISE). La administración de los cuestionarios se debería realizar tras la evaluación de los aprendizajes, acción que cierra el desarrollo de un ciclo educativo (unidad instruccional, bloque temático, asignatura, etc.), pero antes de que los estudiantes conozcan la calificación obtenida, para que dicha información no influya en sus respuestas. 


\section{Referencias}

Anderson, L. y Burns, R. (1989). Research in classrooms. Oxford: Pergamon Press.

Atkinson, J. W. (1964). An introduction to motivation. Princeton, NJ: Van Nostrand.

Beltrán, J. (1993). Procesos, estrategias y técnicas de aprendizaje. Madrid: Síntesis Psicología.

Beltrán, J. (1998). Estrategias de aprendizaje. En J. Beltrán y C. Genovard (Eds.): Psicología de la Instrucción I. Variables y procesos básicos (pp. 383-428). Madrid: Síntesis Psicología.

Boekaerts, M. (1999). Motivated learning: Studying student situation transactional units. European Journal of Psychology of Education, 14(1), 41-55.

Biggs, J. B. (1978). Individual and Group Differences in Study Processes. British Journal of Educational Psychology, 48(3), 266-79.

Cochram-Smith y Lytle (1993). Inside outside: Teacher research and knowledge. New York: Teachers College Press.

Deci, E., y Ryan, R. (1985). Intrinsic motivation and self-determination in human behavior. New York: Plenum.

Cole, A. L. y Knowles, J. G. (2000). Researching teaching: Exploring teacher development through reflective inquiry. Boston: Allyn and Bacon.

De la Fuente, J. (2011). Implications for the DEDEPRO Model for Interactive Analysis of the Teaching-Learning Process in Higher Education. In R. Teixeira (Ed.), Higher Education in a State of Crisis (pp. 205-222). New York: Nova Science Publisher Inc.

De la Fuente, J. y Justicia, F. (2007). The DEDEPRO Model for Regulating Teaching and Learning: recent advances. Electronic Journal of Research in Educational Psychology, $5(3), 535-564$.

Descals, A. (1996). El proceso E/A universitario: estudio intensivo de la situación educativa en Psicología de la Educación( Tesis de licenciatura no publicada). Facultad de Psicología, Universidad de Valencia (Estudi General), Valencia.

Doménech, F. (1991). Aproximación experimental a la Situación Educativa a partir del M.I.S.E. (Área de Ciencias Sociales) ( Tesis de Licenciatura no publicada). Facultad de Psicología, Universidad de Valencia (Estudi General), Valencia. 
Doménech, F. (1995). Estudio empírico de la situación educativa desde el MISE: una aproximación diferencial y estructural (Tesis doctoral no publicada). Facultad de Psicología, Universidad de Valencia (Estudi General), Valencia.

Doménech, F. (2006). Testing an instructional model in a university educational setting from the student's perspective. Learning \& Instruction, 16(5), 450-466.

Doménech, F. (2011a). Evaluar e investigar en la situación educativa universitaria. Un nuevo enfoque desde el Espacio Europeo de Educación Superior. Castellón: Publicaciones de la Universitat Jaume I, Universitas 32.

Doménech, F. (2011b). Examinando la viabilidad de un modelo instruccional: un estudio preliminar desde la perspectiva del estudiante. Comunicación presentada en el VI Congreso Internacional de Psicología y Educación celebrado en Valladolid los días 29-30-31 de marzo y 1 de abril de 2011.

Doménech, F. (2012). Psicología educativa: su aplicación al contexto de la clase. Castellón: Publicacions de la Universitat Jaume I, Psique, 13.

Doménech, F., y Descals, A. (2003). Evaluation of the university teaching/learning process for the improvement of quality in higher education. Assessment \& Evaluation in Higher Education, 28(2), 166-177.

Doyle, W. (1977). Paradigms for research on teacher effectiveness. In L. Shulman (Ed.), Review of research in education (pp. 163-198). Ithaca, IL: Peacock.

Dunkin, M. J., \& Biddle, B. J. (1974). The study of teaching. New York: Holt, Rinehart and Winston.

Entwistle, N. (1987). A model of the teaching-learning process derived from research on student learning. En Richarson, Eysenck \& Warren-Piper (eds.), Student learning research in education and cognitive psychology (pp. 13-28). London, Open University.

Erickson, F. (1986). Qualitative methods in research on teachig. En Wittrock, M. C. Handbook of Research on Teaching (pp. 119-160). Nueva York: MacMillan..

Feather, N. (ed.) (1982). Expectations and Actions. Hillsdale, NJ: Erlbaum

Fraser, B. (1987). Identifying the salient facets of a model of student learning: A synthesis of metaanalyses. International Journal of Educational Research, 11(2), 187-212.

Fraser, B. (1989). Twenty years of classroom climate work: progress and prospect. Journal of Curriculum studies, 21, 307-327. 
Fraser, B. (1998). Science learning environments: assessment, effects and determinants. In B. J. Fraser \& K. G. Tobin (Eds.), International handbook of science education (pp. 527564). Dordrecht, The Netherlands: Kluver.

Gómez, A. (1993). Estudio de la situación educativa científica desde el EL MISE (Tesis de Licenciatura, no publicada). Dpto. Psicología Evolutiva y de la Educación. Universidad de Valencia (Estudi General), Valencia.

Huitt, W. (2003). A transactional model of the teaching-learning process. Educational Psychology Interactive. Valdosta, GA: Valdosta State University. Retrieved from http://www.edpsycinteractive.org/materials/tchlrnmd.html (Consultado el 22-9-12)

Jay, J. K. (2003). Quality teaching: Reflection as the heart of practice. Lanham, MD: Scarecrow Press.

Larrivee, B. (2000). Transforming teaching practice: Becoming the critically reflective teacher. Reflective Practice, 1(3), 293-307.

Lazarus, R. S. y Folkman, S. (1984). Stress, Appraisal and coping. New York: Springer.

Martínez, F. (1991). Aproximación experimental a la situación educativa a partir del MISE (Área de ciencias experimentales): (Tesis de licenciatura no publicada). Facultad de Psicologia, Universidad de Valencia (Estudi General). Valencia.

Martínez, F. (1995): La alfabetización científica en la formación de personas adultas a partir del MISE: un análisis experimental (Tesis Doctoral no publicada). Facultad de Psicologia, Universidad de Valencia (Estudi General). Valencia.

Osterman, K. P. y Kottkamp, R. B. (1993/2004). Reflective practice for educators: Improving schooling through professional development. Thousand Oaks, CA: Corwin Press.

Pascarella, E. (1985). Students' affective development within the college environment. Journal of Higher Education, 56(6), 640-663.

Pintrich, P. R. (1989). The dynamic interplay of student motivation and cognition in the college classroom. En C. Ames and M. L. Maher (eds.): Advances in motivation and achievement (vol. 6) (pp. 117-160). Greenwich, CT: JAI Press.

Pintrich, P. R. (2000). The role of goal orientation in self-regulated learning. In M. Boekaerts, P. R. Pintrich y M. Zeidner (eds.), The handbook of self-regulation (pp.451-502). San Diego: Academic Press.

Pintrich, P. R., y De Groot, E. V. (1990). Motivational and self-regulated learning components of classroom performance. Journal of Educational Psychology, 82, 33-40.

Pozo, J. I. (1989). Adquisición de estrategias de aprendizaje. Cuadernos de Pedagogía, 175, 8-11. 
Ramsden, P. (1992). Learning to teach in higher education. London: Routledge.

Rivas, F. (1993). Modelo integrado de situación educativa (MISE): una aproximación desde la psicología de la instrucción. En V. Pelechano, (Ed.), Psicología, mitopsicología y postpsicología (pp. 293-338). Valencia: Promolibro.

Rivas, F. (1997). El proceso de enseñanza/aprendizaje en la situación educativa. Barcelona: Ariel, Psicología.

Rivas, F. (2003). El proceso de enseñanzalaprendizaje en la Situación Educativa. (2 edición). Barcelona: Ariel, Psicología.

Rivas, F. y Descals, A. (2000): Modelos de instrucción universitaria: revisión y aportaciones. En J. N. García Sanchez (Ed.). De la Psicología de la Instrucción a las necesidades curriculares (pp. 191-199). Barcelona: Oikos-tau.

Rosales, C. (2000). Evaluar es reflexionar sobre la enseñanza ( $3^{\text {a }}$ edición). Madrid: Narcea

Schön, D. A. (1992). La formación de profesionales reflexivos. Hacia un nuevo diseño de la enseñanza y del aprendizaje de los profesionales. Barcelona: Paidós-MEC.

Stufflebeam, D. L. y Shinkfield, A. J. (1989). Evaluación sistemática: guía teórica y práctica. Barcelona: Paidós MEC. .

Vroom, V.H. (1964). Work and motivation. Nueva York: Wiley and sons.

Waxman, H. (1991). Investigating classroom and school learning environments: a review of recent research and developments in the field. Journal of Classroom Interaction, 26(2), 1-4.

Zeichner, K. M. (1993). El maestro como profesional reflexivo. Cuadernos de Pedagogía, 220, 44-49.

Zeichner, K. M. y Liston, D. P. (1996). Reflective teaching: An introduction. Mahwah, NJ: Lawrence Erlbaum. 